Check for updates

Cite this: Mater. Adv., 2021, 2, 4370

Received 14th February 2021 Accepted 12th May 2021

DOI: $10.1039 / \mathrm{d} 1 \mathrm{ma} 00131 \mathrm{k}$

rsc.li/materials-advances

\section{All-fiber pyro- and piezo-electric nanogenerator for loT based self-powered health-care monitoring $\dagger$}

\author{
Biswajit Mahanty, (D) ab Sujoy Kumar Ghosh, (D) $\ddagger^{\mathrm{a}}$ Kuntal Maity, ${ }^{a}$ Krittish Roy, ${ }^{a}$ \\ Subrata Sarkar ${ }^{a}$ and Dipankar Mandal (D) *c
}

\begin{abstract}
In this work, an all-fiber pyro- and piezo-electric nanogenerator (PPNG) is designed using multiwall carbon nanotube (MWCNT) doped poly(vinylidene fluoride) (PVDF) electrospun nanofibers as the active layer and an interlocked conducting micro-fiber based electrode for converting both thermal and mechanical energies into useful electrical power. The PPNG generates high electrical throughput (output voltage $\sim 35 \mathrm{~V}$, maximum power density $\sim 34 \mu \mathrm{W} \mathrm{cm}{ }^{-2}$ and power conversion efficiency $\left.\left(\eta_{\text {piezo }}\right) \sim 19.3 \%\right)$ with an ultra-fast response time of $\sim 10 \mathrm{~ms}$. Owing to the higher piezoelectric charge co-efficient $\left(\left|d_{33}\right| \sim 51.3 \mathrm{pC} \mathrm{N} \mathrm{N}^{-1}\right.$ ) and figure of merit (FoM $\approx 5.95 \times 10^{-11} \mathrm{~Pa}^{-1}$ ) of PVDF-MWCNT nanofibers in comparison to the neat PVDF nanofibers $\left(\left|d_{33}\right| \sim 22 \mathrm{pC} \mathrm{N}{ }^{-1}\right.$ and FoM $\approx 9.7 \times 10^{-12} \mathrm{~Pa}^{-1}$ ) the PPNG operates a range of consumer electronic components such as capacitors and light emitting diodes. Furthermore, the electroactive phase content $(\sim 87 \%)$ is improved in the active layer due to the interfacial interaction between the surface charges at from the $\pi$-electron cloud of the MWCNT and $-\mathrm{CH}_{2}$ - dipoles of the PVDF chain. Additionally, the PVDF-MWCNT nanofibers possess fifteen times higher pyroelectric coefficient $\left(\sim 60 \mathrm{nC} \mathrm{m}{ }^{-2} \mathrm{~K}^{-1}\right.$ ) compared to that of neat PVDF nanofibers $\left(4 \mathrm{nC} \mathrm{m}^{-2} \mathrm{~K}^{-1}\right)$. As a result, the PPNG is capable of converting very large temperature fluctuations $(\Delta T \sim 14.30 \mathrm{~K})$ to electrical energy (such as the open-circuit voltage of $250 \mathrm{mV}$ and a short-circuit current of $83 \mathrm{pA}$ ). Besides this, it is capable of detecting very low-level thermal fluctuations (as low as $\Delta T \sim 5.4 \mathrm{~K}$ ) with responsivity of $\sim 1.48 \mathrm{~s}$ and possesses very high mechano-sensitivity $\left(\sim 7.5 \mathrm{~V} \mathrm{kPa}^{-1}\right)$ which makes it feasible for use as a biomedical sensor since the body temperature and bio-mechanical signals (such as breathing temperature, pulse rate, vocal cord vibrations, coughing sound, and so on) have an immense signature of health conditions. As a proof-of-concept, the all-fiber PPNG is employed as a biomedical sensor by integrating with the Internet of Things (IOT) based human health care monitoring system as well as for remote care of infectious diseases (e.g., applicable for pneumonia, COVID-19) by transferring the pulse response, body temperature, coughing and laughing response wirelessly to a smartphone.
\end{abstract}

\section{Introduction}

Recently, the "Internet of Things (IOT)" has gained considerable attention in numerous applications in remote health care monitoring systems, especially in infectious diseases (e.g. pneumonia, COVID-19). ${ }^{1-3}$ An ultra-sensitive pressure-temperature dual functional pressure sensor is the main component of state-ofthe-art IoT based remote health care systems. It is used to collect the physiological signals from different parts of our body and behaves as a transducer by converting the applied force and temperature fluctuations into an electrical signal, or other perceived signal output. ${ }^{4,5}$ These sensors can be adhered to our regularly used textiles or may be attached on the human skin for real-time monitoring of human health, activities and physiological signal measurements. In real-life application the 
textile based self-powered sensor is an ideal candidate as it is ultra-flexible, light weight and conformable to any surface of the body as well as because no external bias is required to operate it. In this scenario, piezoelectric and pyroelectric materials are ideal candidates as they require no external bias to operate. Conventionally, very highly sensitive pressure sensors are operated by a piezo-resistive and capacitive mechanism which needs an external bias electric field. This essential requirement restricts the convenient utilization and integration of the sensors with wireless communication systems. In terms of the mechanical energy harvesting approach triboelectric nanogenerators are ideal candidates for higher output power generation, $^{6}$ but piezoelectric nanogenerators are essential devices for higher stability and lower pressure sensing applications, specifically physiological signal monitoring. To date, despite their brittle (low toughness) and rigid (high stiffness) nature, a large number of inorganic and lead based materials have been applied to fabricate piezo- and pyro-electric nanogenerators. ${ }^{7}$ In contrast the organic polymer based piezoelectric and pyroelectric materials are of particular interest towards electronic skin because of their high compliance and flexibility, light weight nature and biocompatibility. PVDF is one of the most favorable ferroelectric polymer classes. ${ }^{8}$ Among the five crystallographic forms $(\alpha-, \beta-, \gamma-$, $\delta$ - and $\varepsilon$ ), the $\beta$-phase is the most suitable form for piezoelectric and pyroelectric properties of PVDF. There are several well-known methods available to attain high $\beta$-phase content, including the electrical poling method, where a high electric field (typically $100 \mathrm{kV} \mathrm{mm}{ }^{-1}$ ) is applied. ${ }^{9}$ The challenges with electrical poling strategies can be overcome using the electrospinning method where the introduction of the $\beta$-phase and the in situ alignment of dipoles occur concurrently due to the nano-confinement effect in electrospun nanofibers. In addition, the electrospinning process enables the preparation of flexible and ultra-light piezoelectric membranes, making them more applicable in flexible selfpowered sensors for human health detection or monitoring. However, the performance of electrospun PVDF nanofibers remains relatively low due to the lack of suitable device engineering, which leads to a small output power when used in energy harvesting applications. In this scenario, a large number of researchers have used different types of filler, especially MWCNTs, to improve the performance or efficiency of PVDF electrospun fiber based nanogenerators. ${ }^{10-12}$ The addition of MWCNTs to PVDF significantly improved the output response of nanogenerators. From a material point of view, the feasibility of MWCNTs as a filler material lies in its structural appearance, which is made up of different coaxial cylinders composed of a single layer of graphene around the cylindrical inner hole. It is noticed that a huge number of articles have been reported on PVDF nanofiber based nanogenerators, but a temperaturepressure dual functional all-fiber based wearable sensor was hardly reported. The metal foil or metal-coated thin film-based electrodes for charge collection both have limitations with respect to the device lifetime under the prolonged cyclic stress. ${ }^{13}$ There were a few more limitations found which include the poor fatigue resistance causing early failure of the metal foil and a huge mismatch of the Young's modulus of the metal coated electrode and the active thin film. However, due to the prolonged period of application, loss of mechanical integrity and electric connectivity occurred. Now all these issues can be avoided by adopting an efficient and durable all-fiber nanogenerator of a three-layered structure where both piezoand pyro-electric active constituent and electrodes are composed of adaptable and soft fiber arrays.

In this work, a notable enhancement of the electroactive phase content in the electrospun PVDF nanofiber has been attained by the synergistic effect of interfacial interactions of MWCNTs with the PVDF chain and further mechanical stretching in the course of collection of the fiber by the high speed rotating collector. As a result a very high piezoelectric/pyroelectric coefficient, figure of merit and energy harvesting output power with high power conversion efficiency have been achieved. A highly sensitive all-fiber nanogenerator is designed with PVDF-MWCNT nanofibers which could capture very low level temperature fluctuations and possesses very high pressure sensitivity. With such effective performance, an IoT based wireless health care system is developed through which the physiological signals can be transferred to a smart phone, indicating a promising way of real time remote health care monitoring.

\section{Results and discussion}

\section{All-fiber nanogenerator/sensor fabrication strategy}

The copper $(\mathrm{Cu})$-nickel $(\mathrm{Ni})$ plated interlocked micro-fiber based polyester fabric was used as the top and bottom electrodes to fabricate the piezoelectric and pyroelectric nanogenerator (PPNG). Conducting copper wires were attached on both electrodes and finally the three layered structure PPNG was covered with a PDMS layer. The schematic diagram of the all-fiber nanogenerator is shown in Fig. 1a. The generated electrospun nanofibers are shown in Fig. 1a(i). The all-fiber nanogenerator consists of PVDF-MWCNT electrospun nanofibers (Fig. 1a(ii)) sandwiched between interlocked micro-fiber arrays of conducting fabric as charge collecting electrodes (Fig. 1a(iii)). The device was further encapsulated with PDMS for protection from the environment and to ensure a compact structure of the device. The diameter of the interlocked micro-fiber of the conducting electrode was $15 \mu \mathrm{m}$. The excellent flexibility and conformability of the generated electrospun nanofibers to the human finger is shown in Fig. 1b. The digital image of the fabricated original PPNG is shown in Fig. S1 (ESI $\dagger$ ). Thus, in the fabricated PPNG active piezoelectric and pyroelectric component and the electrodes all are composed of fibers.

\section{Structural and electrical properties}

The FE-SEM image with the corresponding histogram profiles of PVDF-MWCNT nanofibers is shown in Fig. 2a. The fiber diameter distribution plot is presented in the inset of Fig. 2a. It is observed that randomly oriented nanofibers are formed and the average diameter of the fibers ranges from 40 to $165 \mathrm{~nm}$, with the maximum number of fibers possessing a diameter of $\sim 85 \mathrm{~nm}$. During the formation of fibers i.e., 


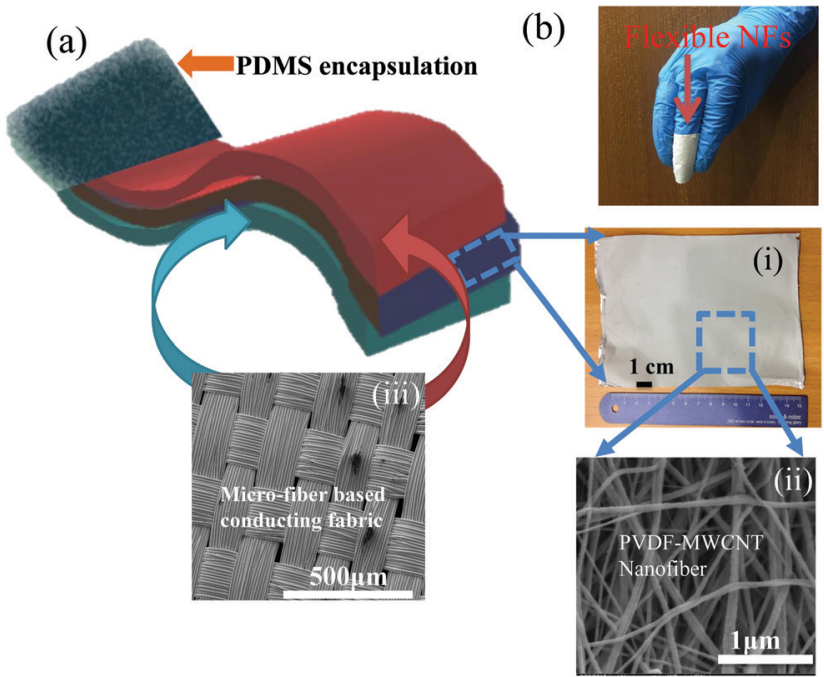

Fig. 1 (a) Schematic of the all-fiber PPNG comprising of (i) a large area PVDF-MWCNT nanofiber mat $(8 \mathrm{~cm} \times 7 \mathrm{~cm}$ ). (ii) FESEM images of the PVDF-MWCNT nanofiber and (iii) micro-fiber based conducting electrode. (b) The excellent flexibility and conformability of PVDF-MWCNT electrospun nanofibers is shown by wrapping them on a human finger.
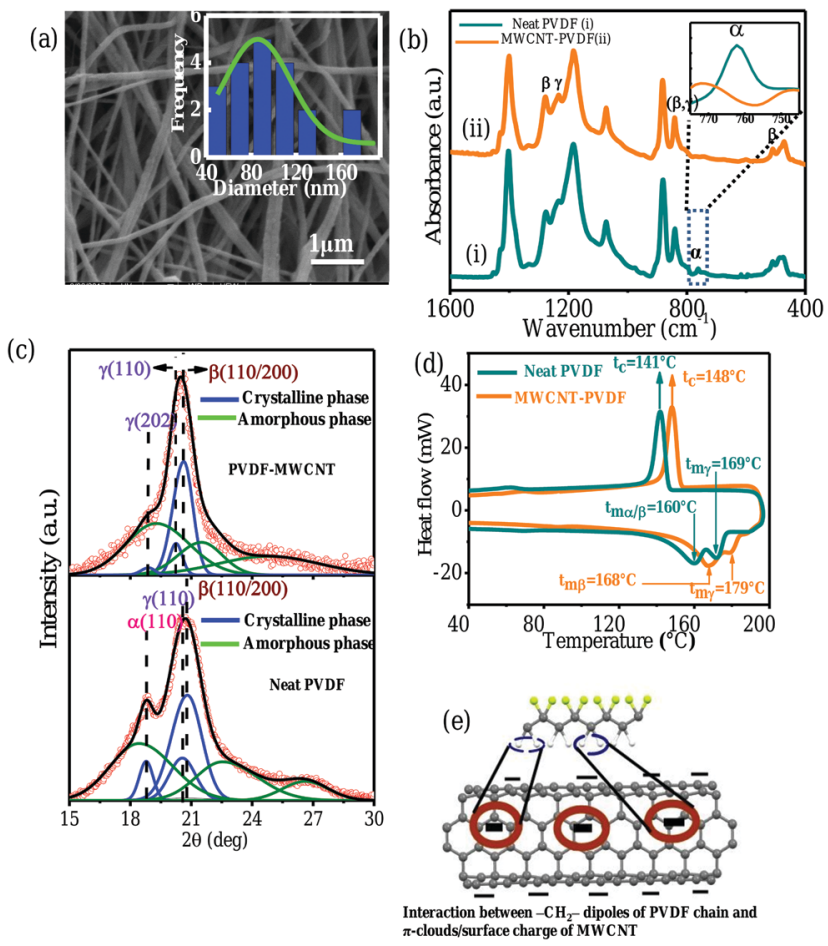

Fig. 2 (a) FE-SEM image and the corresponding fiber diameter distribution plot in the inset, (b) FT-IR spectra of PVDF-MWCNT and neat PVDF nanofibers in the region of $1600-400 \mathrm{~cm}^{-1}$, (c) XRD patterns and their curve deconvolution of PVDF-MWCNT nanofibers and neat PVDF nanofibers, (d) DSC thermograms of PVDF-MWCNT and neat PVDF nanofibers. (e) Schematic of the interaction between $-\mathrm{CH}_{2}$ - dipoles of the PVDF chain and $\pi$-electron clouds/surface charges of MWCNTs.

electrospinning process, the molecular dipoles of PVDF nanofibers are preferentially oriented in the out-of-plane direction since the nanofibers and polymer chains are aligned within the plane of the collector. ${ }^{14}$ This is due to the simultaneous application of the electric field and mechanical stretching leading to polymer jet elongation, whipping and in situ poling. ${ }^{15}$ During electrospinning of the MWCNT mixed PVDF solution, the conductive MWCNTs can produce inductive charges on their surface when the external electric field is applied, which results in a greater Coulomb force during the electrospinning process. This effect helps to improve the piezoelectric and pyroelectric properties of electrospun PVDFMWCNT nanofibers due to the excellent interfacial interaction between the PVDF polymer chain and MWCNT surface charges the nanoscopic scale. Therefore, a synergistic effect occurred between interfacial interaction and mechanical stretching during the collection of fibers by a high speed rotating collector during crystallization of fibers. As a result, the content of electroactive $\beta$-phase was much higher in PVDF-MWCNT nanofibers than in the neat (pure) PVDF nanofibers and this is evident from the FT-IR plot (see Fig. 2b). The FT-IR spectra of PVDF-MWCNT and neat PVDF nanofibers are shown in Fig. 2b, in the region of $1600-400 \mathrm{~cm}^{-1}$. Evidently the neat PVDF nanofibers contain the $\alpha$-phase (non-polar) (see the vibrational band at $762 \mathrm{~cm}^{-1}$ in the inset of Fig. $2 \mathrm{~b}$ ), and $\beta$ - and $\gamma$-(polar) phases (vibrational bands at 1277 and $1234 \mathrm{~cm}^{-1}$ respectively). ${ }^{9,16}$ However, the PVDF-MWCNT nanofibers contain only polar $\beta$ - and $\gamma$-phases and no $\alpha$-phase peak is observed in the spectrum, as seen in the inset of Fig. $2 b$. This implies the conversion of non-polar phases ( $\alpha$-phase) into polar phases ( $\beta$ - and $\gamma$-phases) in PVDF-MWCNT nanofibers in comparison to neat PVDF nanofibers by the electrospinning process. Eventually, it is found that both the nanofibers having $841 \mathrm{~cm}^{-1}$ vibrational band, which presenting the coexistence of $\beta$ - and $\gamma$-phases.

Assuming that the infrared absorption follows the BeerLambert law, the relative proportion of electroactive $\beta$ - and $\gamma$-phases (defined as $F_{\mathrm{EA}}$ ) is calculated using eqn (1) as

$$
F_{\mathrm{EA}}=\frac{A_{841}}{\left(\frac{K_{841}}{K_{762}}\right) A_{762}+A_{841}} \times 100 \%
$$

where $A_{762}$ and $A_{841}$ represent the absorbency at 762 and $841 \mathrm{~cm}^{-1}$ respectively, and $K_{762}=6.1 \times 10^{4} \mathrm{~cm}^{2} \mathrm{~mol}^{-1}$ and $K_{841}=7.7 \times 10^{4} \mathrm{~cm}^{2} \mathrm{~mol}^{-1}$ represent the absorbance coefficient corresponding to wave numbers. ${ }^{9,17,18}$ Therefore, the calculated $F_{\mathrm{EA}}$ of neat PVDF nanofibers is $F_{\mathrm{EA}} \sim 67 \%$, whereas in the case of PVDF-MWCNT nanofibers, $F_{\mathrm{EA}} \sim 87 \%$.

Additionally, Fig. 2c shows the curve-deconvoluted XRD patterns of the PVDF-MWCNT and neat PVDF nanofibers. As evident, the diffraction peak at $2 \theta$ of $19.2^{\circ}$ in neat PVDF nanofibers corresponds to the reflection of the $\alpha$-crystalline phase (110) and $2 \theta$ of $20.8^{\circ}$ corresponds to the cumulative peak of the $\beta$ - and $\gamma$-crystalline phase (110/200). Although the electrospinning technique is a very good option to induce all-trans configuration ( $\beta$-phase) in the PVDF crystal structure, the $\alpha$-phase still exists in the neat PVDF nanofibers, which can be understood from the XRD and FT-IR analysis. The $\alpha$-crystalline 
phase is fully diminished in the composite fibers (Fig. 2b and c), which is due to the addition of MWCNTs in the PVDF matrix. The conversion of the non-polar to polar phase occurs due to two factors:

1. Simultaneous poling and stretching during the electrospinning process.

2. The interfacial interaction present between the surface charge/ $\pi$-cloud of MWCNTs and $-\mathrm{CH}_{2}-$ dipoles of the PVDF chain.

The degree of crystallinity $\left(\chi_{c}\right)$ is calculated from

$$
\chi_{\mathrm{c}}=\frac{\sum A_{\mathrm{cr}}}{\sum A_{\mathrm{cr}}+\sum A_{\mathrm{amr}}} \times 100 \%,
$$

where $\sum A_{\mathrm{cr}}$ and $\sum A_{\mathrm{amr}}$ are the summation of the integral area of crystalline peaks and amorphous halo respectively. Due to the addition of MWCNTs, the total crystallinity of PVDF-MWCNT nanofibers is higher (59\%) than that of neat PVDF (53\%). The crystallization along with thermal stabilization of the $\beta$-phase in PVDF-MWCNTs was evaluated by differential scanning calorimetry (DSC) study, as shown in Fig. 2 d.

It can be seen that both the melting peaks $\left(t_{\mathrm{m}}\right)$ of the two types of nanofibers indicate the existence of $\beta$-and $\gamma$-phases. The endothermic melting peak of the $\beta$ - and $\gamma$-phases is found in PVDF-MWCNT nanofibers at $\sim 168{ }^{\circ} \mathrm{C}$ and $\sim 179{ }^{\circ} \mathrm{C}$ respectively. ${ }^{16,19-21}$ For the neat PVDF nanofibers, it is found that the melting peak of $\alpha / \beta$-phases is at $\sim 160{ }^{\circ} \mathrm{C} .{ }^{16,19-21}$ As a result, both $\beta$ - and $\gamma$-phases are considered more stable thermally in the PVDF-MWCNT nanofibers with respect to neat PVDF nanofibers. As a consequence, the crystallization temperature $\left(t_{\mathrm{c}}\right)$ of PVDF-MWCNT nanofibers $\left(\sim 148{ }^{\circ} \mathrm{C}\right)$ was also enhanced with respect to the neat PVDF $\left(\sim 141^{\circ} \mathrm{C}\right)$. This is due to MWCNTs acting as a nucleating agent and hindering the movement of polymer chain segments. Moreover, the induced polar $\beta$-phase is stabilised during rapid crystallization and not relaxed back to the non-polar $\alpha$-phase via thermal motion. As a result, the formation of the crystalline $\beta$-phase is irreversible in the PVDF-MWCNT nanofibers and not relaxed back to the $\alpha$-phase via thermal motion. ${ }^{22}$ It is noteworthy that in the case of the free standing film sample containing the gamma phase, the melting point is at a relatively much higher side. ${ }^{19}$ In contrast, the electrospun samples act a bit differently which may be because of air-permeable spongy like behaviour that leads to lower melting temperature compared to that of the right film specimen. In addition, from the DSC data the total degree of crystallinity $\left(\chi_{\mathrm{ct}}\right)$ of the nanofibers was calculated using

$$
\chi_{\mathrm{ct}}=\frac{\Delta H_{\mathrm{m}}}{(1-\phi) \Delta H_{\mathrm{m}}^{0}}
$$

where $\Delta H_{\mathrm{m}}$ represents the melting enthalpy, $\Delta H_{\mathrm{m}}^{0}$ is the melting enthalpy of the $100 \%$ crystalline PVDF $\left(\sim 104.7 \mathrm{~J} \mathrm{~g}^{-1}\right)$ and $\varphi$ is the weight fraction of MWCNTs. ${ }^{22} \chi_{\mathrm{ct}}=40 \%$ and 33\% for PVDFMWCNT and neat PVDF nanofibers respectively. Overall, the improved electroactive phase content, improved degree of crystallinity and higher thermal stabilization are the result of the synergistic effect. Therefore, it is concluded that the degree of crystallinity of PVDF-MWCNT nanofibers is improved due to the presence of MWCNTs. From the material point of view, it is very evident that PVDF-MWCNT nanofibers are an excellent material for application in piezo- and pyro-electric nanogenerators. Of particular interest, the interfacial interaction between the MWCNT NPs and PVDF dipoles is schematically shown in Fig. 2e. Here, MWCNTs mainly act as a nucleating agent. During the electrospinning process, charge is accumulated at the interfacial boundaries between MWCNTs and non-polar $\alpha$-phase and ferroelectric $\beta$-phase. ${ }^{23}$ In addition, there might exist dipolar intermolecular interactions between the $\pi$-cloud of MWCNTs and $-\mathrm{CH}_{2}-$ dipoles of the PVDF chain. So, the inter-conversion of $\alpha$ - and $\beta$-phases near the MWCNT surface can be described by the charge accumulation at the interphase and dipolar interactions. Moreover, the induced polar $\beta$-phase is stabilised during rapid crystallization and not relaxed back to the non-polar $\alpha$-phase via any thermal motion.

\section{Pyroelectric energy harvesting}

The harvesting of thermal energy from the all-fiber PPNG is obtained by the pyroelectric effect. The temperature fluctuations are maintained using an IR light source placed on the top surface of the PPNG. The pyroelectric energy harvesting performance of the PPNG is shown in Fig. 3 in terms of the measurement of the open circuit voltage $\left(V_{\mathrm{oc}}\right)$ under different temperature fluctuations of $\Delta T \sim 14.3 \mathrm{~K}$ (Fig. 3a) and $\Delta T \sim$ $5.4 \mathrm{~K}$ (Fig. 3c) as well as the corresponding short circuit current $\left(I_{\mathrm{sc}}\right)$ with the temperature gradient $(\mathrm{d} T / \mathrm{d} t)$ in Fig. $3 \mathrm{~b}$ and $\mathrm{d}$ respectively. It is noteworthy to mention that $I_{\mathrm{sc}}$ and $V_{\mathrm{oc}}$ are $71 \mathrm{pA}$ and $250 \mathrm{mV}$ (approx.) respectively when $\Delta T \sim 14.3 \mathrm{~K}$ and $\mathrm{d} T / \mathrm{d} t=2.15 \mathrm{~K} \mathrm{~s}^{-1}$ under the temperature switching frequency of $0.01 \mathrm{~Hz}$ which is much higher than that of the device made from neat PVDF. $^{24}$ When the switching frequency changes from $0.01 \mathrm{~Hz}$ to $0.1 \mathrm{~Hz}\left(\Delta T \sim 5.4 \mathrm{~K}\right.$ and $\left.\mathrm{d} T / \mathrm{d} t=1.72 \mathrm{~K} \mathrm{~s}^{-1}\right) I_{\mathrm{sc}}$ and $V_{\text {oc }}$ are $83 \mathrm{pA}$ and $135 \mathrm{mV}$ (approx.) respectively as shown in Fig. $3 \mathrm{c}$ and $\mathrm{d}$. The short circuit current $I_{\mathrm{sc}}$ can be expressed as

$$
I_{\mathrm{sc}}=A \frac{\mathrm{d} P}{\mathrm{~d} T} \frac{\mathrm{d} T}{\mathrm{~d} t}=A p \frac{\mathrm{d} T}{\mathrm{~d} t}
$$

where $A$ is the surface area $\left(\mathrm{m}^{2}\right), \frac{\mathrm{d} T}{\mathrm{~d} t}$ is the rate of temperature change $\left(\mathrm{K} \mathrm{s}^{-1}\right)$ and $p$ is the pyroelectric coefficient $\left(\mathrm{nC} \mathrm{m}^{-2} \mathrm{~K}^{-1}\right){ }^{24-26}$ This enhancement of $I_{\mathrm{sc}}$ was accomplished by a thermally induced piezoelectric coupling effect, as the output performance is proportional to $\Delta T$ and $\mathrm{d} T / \mathrm{d} t$. The available maximum power density $(P)$ of the PPNG is estimated to be $\sim 12 \mathrm{nW} \mathrm{m}^{-2}$ when the temperature difference between the electrodes is $\Delta T \sim 14.3 \mathrm{~K}$. Using eqn (4), the estimated pyroelectric coefficient $(p)$ of the PPNG is $60 \mathrm{nC} \mathrm{m}^{-2} \mathrm{~K}^{-1}$, which is almost fifteen times higher than that of the neat PVDF fiber $\left(4 \mathrm{nC} \mathrm{m}^{-2} \mathrm{~K}^{-1}\right){ }^{24} I_{\mathrm{sc}}$ and $V_{\mathrm{oc}}$ are found to be linearly proportional to $\mathrm{d} T / \mathrm{d} t$ and $\Delta T$ profiles respectively (Fig. 3e and f) in agreement with the theoretical paradigm. These linear characteristics suggest the potential of the PPNG as a self-powered pyroelectric temperature sensor for future practical applications especially in various infectious diseases. Fig. $3 g$ shows the expanded view of the positive current pulse signal of the 

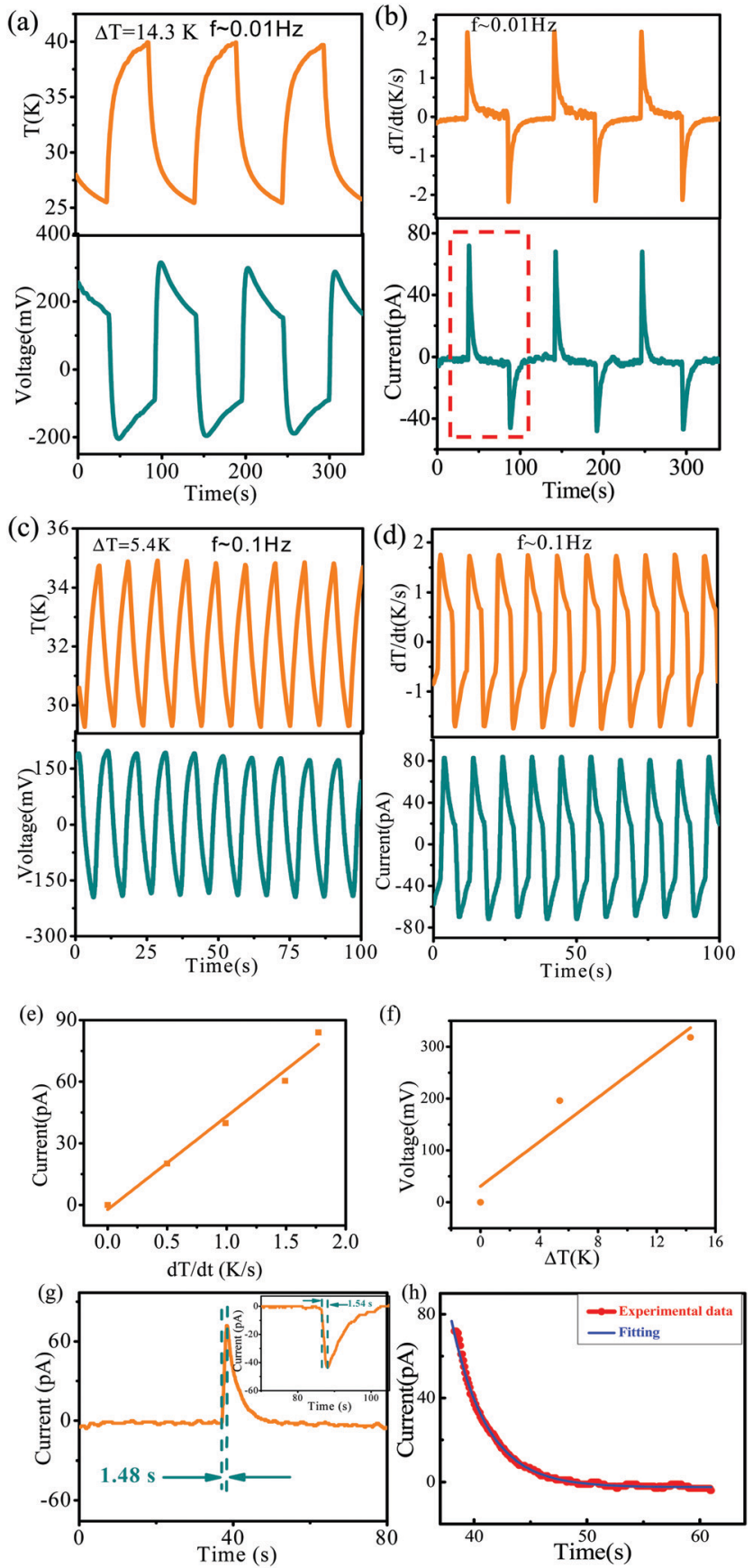

Fig. 3 Measurement of output open circuit voltage under temperature fluctuations of (a) $\Delta T \sim 14.3 \mathrm{~K}$ and (c) $\Delta T \sim 5.4 \mathrm{~K}$ and the corresponding output short circuit current with the variation of rate of temperature $(\mathrm{d} T / \mathrm{d} t)$ in (b) and (d) respectively. (e) Variation in output current as a function of rate of change in temperature. (f) Output voltage as a function of temperature difference. (g) The expanded view of the positive output current signal of the marked cycle in (b) and the negative pulse signal in the inset. (h) The expanded view of the current $\left(I_{\mathrm{sc}}\right)$ vs. time $(T)$ curve of the marked cycle in (b) when the source of heat was removed.

rectangular red dotted marked cycle of Fig. 3b. It has been observed that the current increases from zero to its peak value within $\sim 1.48 \mathrm{~s}$ in the positive side while in the negative side it takes $\sim 1.54 \mathrm{~s}$ (inset of Fig. $3 \mathrm{~g}$ ). As soon as the heat source is removed, the positive output current is returned back to a minimum value. Fig. 3 h represents the " $I_{\mathrm{sc}} v s$. $T$ " curve where it is observed that the current pulse is decreasing exponentially with a time constant value of $2.60 \mathrm{~s}$. This decaying process corresponds to a temperature reduction of the PPNG. The value of the reset time for the PPNG is estimated as the time required to recover to $1 / e$ or $(37 \%)$ and is approximately $2.60 \mathrm{~s}$. So, it is noteworthy to mention that the PPNG can be used as a good temperature sensor due to its reduced response time and reset time compared to that of the previously reported thermoelectric sensor. $^{27}$

The better pyroelectric performance of PVDF-MWCNT nanofibers is ascribed to the infrared transparency $(\sim 5 \%)^{28}$ and improved absorption property of electromagnetic energy by MWCNTs which generates rapid heating in the nanofibers. When the device is exposed to heat (i.e. $\mathrm{d} T / \mathrm{d} t>0$ ) the short circuit current $\left(I_{\mathrm{sc}}\right)$ reaches its positive peak and a negative peak through the cooling process (i.e. $\mathrm{d} T / \mathrm{d} t<0$ ). This happens because of the decrement of the degree of spontaneous polarization at the time of the heating process, which forces the surface charges to flow within the two polar surfaces. The current flows in the opposite direction because of the attractive force of the free charges to the polar surfaces when the device is subsequently cooled. ${ }^{25}$ From the output voltage and current graph, it is very evident that the output current follows the temperature gradient profile (as $I_{\mathrm{sc}} \propto \mathrm{d} T / \mathrm{d} t$ ) and the output voltage follows the thermal fluctuation $\left(V_{\mathrm{oc}} \propto \Delta T\right)$ profile. A summary or comparison of device materials, electrode materials, output performance and pyroelectric coefficient of the PPNG with the reported pyroelectric nanogenerators is given in Table S1 (ESI $\dagger$ ). It has been observed that in spite of the lower pyroelectric co-efficient of PVDF-MWCNT nanofibers in many cases the output performance, in terms of output voltage and current, is much higher than that of previous reports.

\section{Piezoelectric energy harvesting}

To demonstrate the application of the materials, we have fabricated a PVDF-MWCNT based all-fiber nanogenerator/ energy harvester (PPNG). Electrical characterization of the PPNG is shown in Fig. 4a-f and repetitive finger striking over the PPNG surface from a height of $5 \mathrm{~cm}$ is undertaken with different magnitudes of compressive stress $\left(\sigma_{\mathrm{a}}=2,4\right.$ and $\left.6 \mathrm{kPa}\right)$, as shown in Fig. 4a. As a result, the PPNG generates open circuit output voltages $\left(V_{\text {oc }}\right)$ of 11,20 and $35 \mathrm{~V}$ respectively, showing more than $90 \%$ improvement in output voltage under a compressive stress $\sigma_{\mathrm{a}}=2 \mathrm{kPa}$ over the neat PVDF based nanogenerator (see Fig. S2, ESI $\dagger$ ). In addition, the press and release response of the single peak from the PPNG is shown in the right lower inset of Fig. $4 \mathrm{a}$ and it is found that the PPNG shows an ultra-fast response time of $\sim 10 \mathrm{~ms}$. It is important to note that the generated $V_{\text {oc }}$ varies almost linearly with the increase of $\sigma_{\mathrm{a}}$ (right upper inset of Fig. 4a) which is consistent with the piezoelectric theory. As a result, the mechanosensitivity $\left(S_{\mathrm{M}}\right)$, where $S_{\mathrm{M}}=\left(\Delta V_{\mathrm{oc}} / \Delta F\right)$, has been determined to quantify the dynamic mechanical stimuli sensing ability where $\Delta V_{\text {oc }}$ and $\Delta F$ are the differences of the output voltage and force 

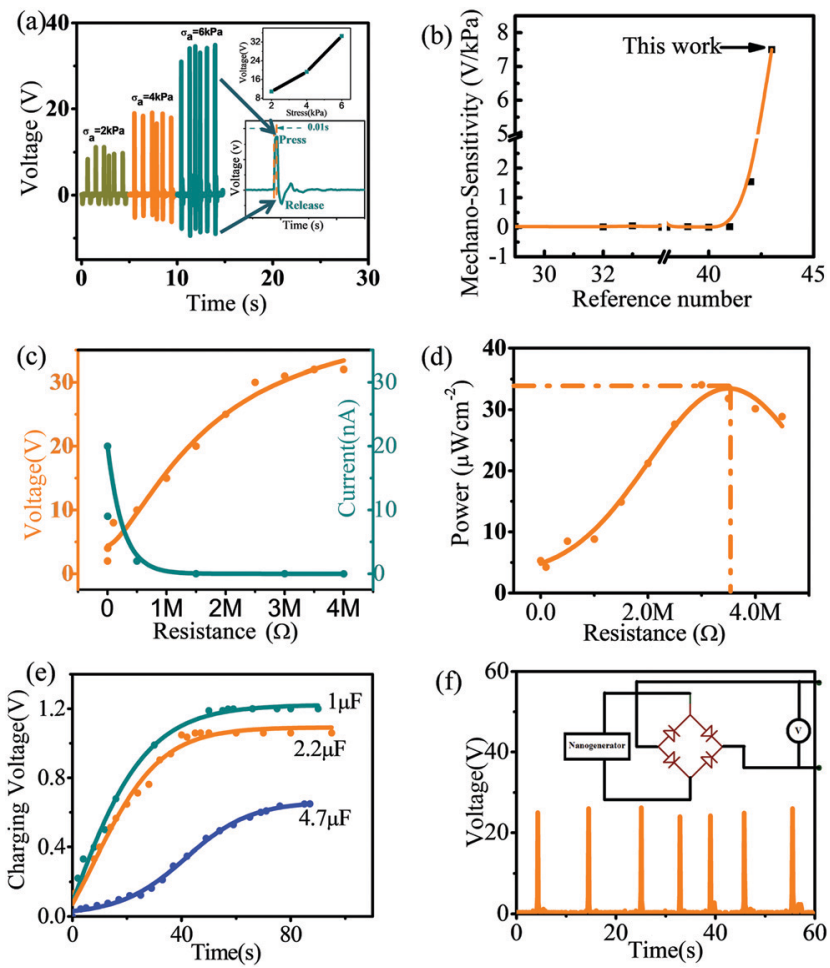

Fig. 4 Energy harvesting characterization of nanogenerators. (a) Pressure dependent output open circuit voltage $\left(V_{\text {oc }}\right)$ of the PPNG, $(b)$ mechanosensitivity of reported piezoelectric based pressure sensors. The abscissa indicates cited references. (c) The variation of output voltage and current with external load resistances, (d) the output power as a function of external load resistances, (e) capacitor charging behavior with different capacitor values, (f) output open circuit rectified voltage response with a bridge rectifier circuit.

respectively. ${ }^{12,22}$ The average mechano-sensitivity $\left(S_{\mathrm{M}}\right)$ of the PPNG is $\sim 7.5 \mathrm{~V} \mathrm{kPa}^{-1}$, which is higher than that of the earlier published high performance nanogenerators. ${ }^{18,24,29-31}$ A detailed comparison plot of piezoelectric mechano-sensitivity with cited references is provided in Fig. 4b. ${ }^{32-37}$ The horizontal line is used to guide the eyes whereas the vertical line indicates the improved value of this work. It is very prominent that the sensitivity of our device is much improved over previous research and is very much suitable for healthcare monitoring applications where sensitivity is the major factor. The superior energy harvesting performance of the PPNG is attributed to the higher piezoelectric charge coefficient $d_{33}=g_{33} \varepsilon_{0} \varepsilon_{\mathrm{r}}=51.3 \mathrm{pC} \mathrm{N}^{-1}$, where $g_{33}$ is the piezoelectric voltage coefficient, $\varepsilon_{0}$ is the permittivity of free space $\left(\sim 8.85 \times 10^{-12} \mathrm{~F} \mathrm{~m}^{-1}\right)$ and $\varepsilon_{\mathrm{r}} \approx 5$ at $1 \mathrm{kHz}$ is the dielectric constant of PVDF-MWCNT nanofibres. ${ }^{38,39}$ The estimated piezoelectric voltage coefficient was found as $g_{33} \sim \frac{V_{\mathrm{oc}}}{\sigma_{\mathrm{a}} \times t}=$ $1.16 \mathrm{~V} \mathrm{~m} \mathrm{~N}^{-1}$, where $\sigma_{\mathrm{a}}=6 \mathrm{kPa}$ is the stress magnitude and $t$ is the thickness of the PPNG. ${ }^{39}$ Thus, PVDF-MWCNT nanofibers exhibit a superior piezoelectric figure of merit $\left(\mathrm{FoM} \approx g_{33} \cdot d_{33} \approx\right.$ $\left.5.95 \times 10^{-11} \mathrm{~Pa}^{-1}\right)$ to those of the neat PVDF nanofibers $\left(d_{33} \sim\right.$ $22 \mathrm{pC} \mathrm{N}^{-1}$ and FoM $\approx 9.7 \times 10^{-12} \mathrm{~Pa}^{-1}$ ) and other various state-of-the-art PVDF-based nanofibres. ${ }^{22}$ The improvement in the piezoelectric coefficient with the addition of MWCNTs is due to the high amount of the $\beta$-phase in the PVDF. We propose that the presence of MWCNTs alters the orientation of the dipoles in the PVDF nanofibers. The incorporation of fillers causes PVDF to swell leading to the formation of extended chain conformation of PVDF around the fillers. Furthermore, adding MWCNTs to reinforce PVDF fibers can enhance the crystallinity of the $\beta$-phase enhancing piezoelectric properties. The $d_{33}$ value of the PVDF-MWCNT nanofibers is found to be significantly greater than those of various PVDF-based nanofibres; a comparison of nanofibres is given in Table S2 (ESI $\dagger$ ). To explore the PPNG as an energy harvesting power source, we have demonstrated in some other way where the output voltages were measured as a function of the external load resistors $\left(R_{\mathrm{L}}\right)$ (Fig. $4 \mathrm{c}$ ) and it is obvious that the instantaneous output voltage drop across the resistive load steadily builds up as the load resistance increases and remains constant at infinitely large resistance ( $4 \mathrm{M} \Omega)$. In addition, in the same figure (Fig. 4c) the variation of the load current with the external load resistors is shown. The instantaneous output power density $(P)$ of the PPNG was calculated as

$$
P=\frac{1}{A} \frac{V_{\mathrm{L}}^{2}}{R_{\mathrm{L}}}
$$

where $A$ is the effective contact area and $V_{\mathrm{L}}$ is the voltage drop across load resistance $R_{\mathrm{L}}$. It is obvious from Fig. $4 \mathrm{~d}$ that the available instantaneous output power density $(P)$ of $34 \mu \mathrm{W} \mathrm{cm}{ }^{-2}$ at a load resistance $\left(R_{\mathrm{L}}\right)$ of 3.4 $\mathrm{M} \Omega$ has been accomplished and it is noted that this high output power density is enough to make on few $(\sim 10)$ blue LEDs when subject to a mechanical pressure without using a storage system. In the interest of demonstrating the practical application of the all-fiber PPNG, the PPNG was connected to external capacitors $(1 \mu \mathrm{F}, 2.2 \mu \mathrm{F}$ and $4.7 \mu \mathrm{F})$ through a full-wave bridge rectifier circuit, and charging up capacitors under periodic human finger imparting stress amplitude of $6 \mathrm{kPa}$ and capacitor charging behavior is shown in Fig. 4e. It is important to note that the PPNG was able to charge up capacitors of several capacitances (such as $1 \mu \mathrm{F}, 2.2 \mu \mathrm{F}$ and $4.7 \mu \mathrm{F}$ ) to $1.18 \mathrm{~V}$, $1.07 \mathrm{~V}$ and $0.65 \mathrm{~V}$ in $55 \mathrm{~s}, 60 \mathrm{~s}$ and $78 \mathrm{~s}$ respectively. The value of the time constant $(\tau)$ for all the capacitors is calculated as the time required to recover to $(1-1 / e)$ or $(63 \%)$ of their steady state voltage and is approximately $19 \mathrm{~s}, 20 \mathrm{~s}$ and $48 \mathrm{~s}$ respectively which ensures the rapid energy supply ability of the PPNG. The power $(P)$ stored in the capacitor is calculated as

$$
P=\frac{C V_{\mathrm{S}}^{2}}{2 t},
$$

where $C$ is the capacitance of the charging capacitor, $V_{\mathrm{S}}$ is the saturation voltage, and $t$ is the time required to reach the saturation voltage. The estimated powers stored in the capacitors are found to be $0.013 \mu \mathrm{W}, 0.021 \mu \mathrm{W}$ and $0.013 \mu \mathrm{W}$ respectively. Fig. $4 \mathrm{f}$ shows the rectified $V_{\mathrm{oc}}$ under $6 \mathrm{kPa}$ of compressive stress using a bridge rectifier circuit (right upper inset) which was used to charge up the capacitors. Larger is the deformation in the nanofibers when the applied compressive stress is higher and as a result an enhancement of the piezopotential is found in the PPNG. More importantly, the long term stability in the output voltage generation from the PPNG is shown in Fig. S3 (ESI $\dagger$ ). 
The instantaneous piezoelectric energy conversion efficiency $\left(\eta_{\text {piezo }}\right)$ of the PPNG is defined as the ratio of the generated output electrical energy (by the instantaneous compressive axial stress and the residual mechanical vibration after the impacting $)\left(E_{\text {elec }}\right)$ to the input mechanical energy $\left(E_{\text {mec }}\right)$. The optimum resistance (3.4 M $\Omega$ ) was connected with the PPNG to derive optimized output performance and the output voltage per cycle was measured instantaneously, as shown in Fig. 4c and Fig. S4 (ESI†).

$$
E_{\text {elec }}=\int_{0}^{t} \frac{V(t)^{2}}{R} \mathrm{~d} t,
$$

The evaluated output energy, $E_{\text {elec }}$ was $8.33 \times 10^{-6} \mathrm{~J}$.

To determine the input mechanical energy $\left(E_{\text {mec }}\right)$ we have to consider the total axial deformation of the PPNG under $6 \mathrm{kPa}$ stress as $\Delta L=\varepsilon \times L$. Here, $\varepsilon$ is the developed axial strain and $L$ is the total thickness of the device. The generated axial strain is given by $\varepsilon \approx \frac{\sigma_{\mathrm{a}}}{Y}=0.13$ where $Y \sim 0.31 \mathrm{GPa}$ is the Young's modulus of the PVDF-MWCNT nanofibres. ${ }^{40}$ Thus, the total input mechanical energy per cycle is calculated as $E_{\text {mec }}=F \times \Delta L=$ $0.63 \times 10^{-6} \mathrm{~J}$, where $F$ is the applied force.

The instantaneous piezoelectric energy conversion efficiency of the PPNG can now be written as $\eta_{\text {piezo }}=\frac{E_{\text {elec }}}{E_{\text {mec }}} \times 100=19.3 \%$.

Finally it is found that the instantaneous piezoelectric energy conversion efficiency of the PPNG is superior to that of previously reported works. ${ }^{22,41} \mathrm{~A}$ comparison of device materials, electrode materials and percentage of piezoelectric energy conversion efficiency $\left(\% \eta_{\text {piezo }}\right)$ of the PPNG with the reported nanogenerators is shown in Table S3 (ESI $\dagger$ ) where in many cases the $\% \eta_{\text {piezo }}$ of our developed device is much higher than that of previous works.

A summary or comparison of device materials, electrode materials, and output performance of the PPNG with the reported nanogenerators is shown in Table S4 (ESI $\dagger$ ) where in many cases the output power of our developed device is much higher than that of previous works. Importantly, previous research on MWCNT reinforced PVDF composites didn't explore the temperature and pressure dual functionality in the device form which is one of the main focus areas of this research. Table S5 (ESI $\dagger$ ) clearly indicates the uniqueness of this work in terms of both thermal and mechanical energy harvesting over previous research.

\section{Healthcare monitoring system}

In this section the PPNG is being used as a self-powered sensor. Here the PPNG was attached on the human skin to detect slight movement of the palm, wrist, arm muscle, leg muscle, and throat (coughing and laughing situation) and different voltage responses are shown in Fig. 5a-f and the corresponding digital picture during the test is shown in the inset. The corresponding positive and negative voltages generated by palm and wrist bending and releasing are shown in Fig. $5 \mathrm{a}$ and $\mathrm{b}$ which are mainly caused by the deformation of epidermis during wrist movements. Fig. $5 \mathrm{c}$ and $\mathrm{d}$ show the output voltage response to
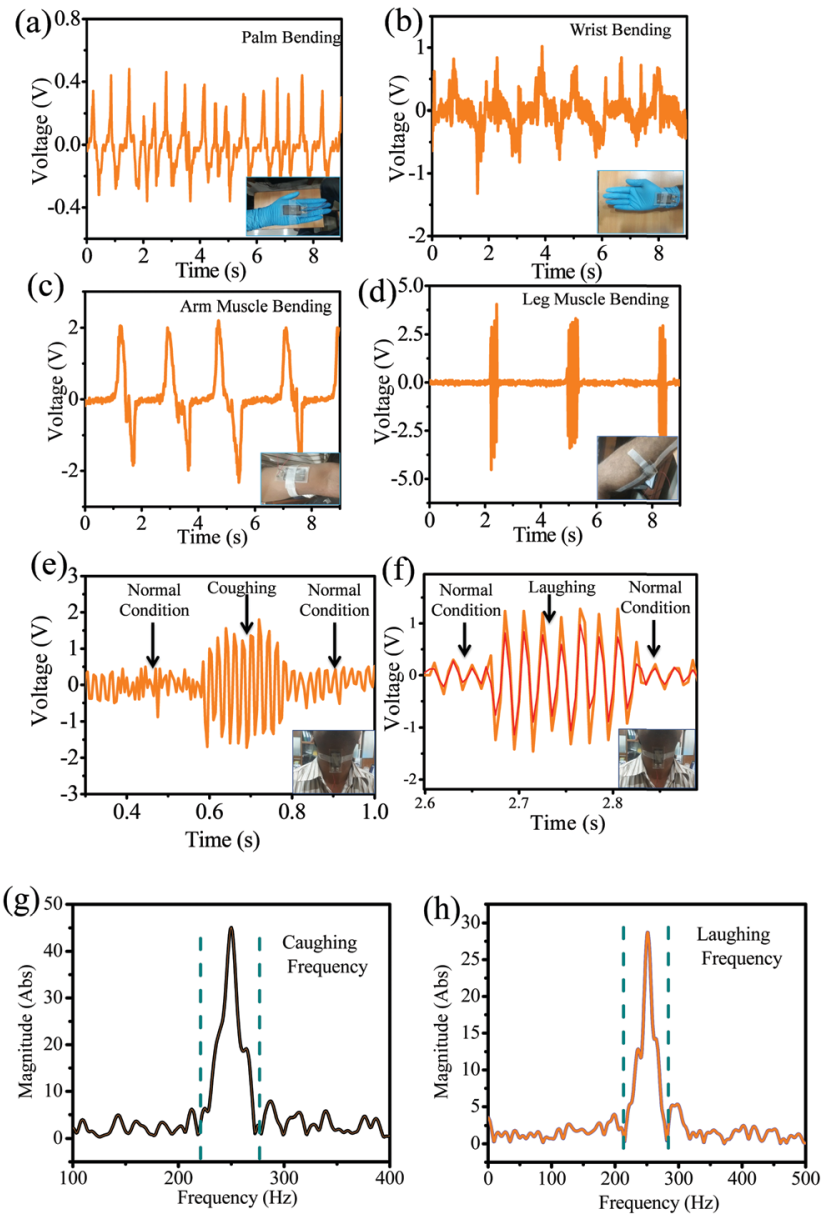

Fig. 5 PPNG for detecting human motions as a sensor. Open circuit voltages when the PPNG is attached on the human skin to detect (a) palm bending, (b) wrist motion, (c) arm muscle bending, (d) leg muscle bending, (e) coughing signal and (f) laughing signal. The insets show the corresponding photography images for the tests, (g) FFT treated cough acoustic frequency spectrum and (h) laughing acoustic frequency spectrum.

the arm and leg muscle movement. Furthermore, the PPNG could transduce the vocal cord vibrations (Fig. 5e and f) due to the coughing and laughing situation when the device is attached to the throat. Fig. $5 \mathrm{~g}$ and $\mathrm{h}$ present the Fast Fourier Transform (FFT) of the coughing and laughing signal (shown in Fig. 5e and f). The FFT treated cough acoustic frequency spectrum 200-380 $\mathrm{Hz}$ and laughing acoustic frequency spectrum $126-424 \mathrm{~Hz}$ are presented respectively which are in good agreement with the coughing and laughing sound of healthy people..$^{22,42,43}$ Therefore, the PPNG can be used as a self-powered sensor to monitor and separate virus infected patients from normal humans. It is noteworthy to mention that the PPNG can be used for self-powered human-motion detection as it exhibited a good repeatability and high responsivity to strain variation caused by the joint/muscle movements without external power supply. Also this sensory information from the skin attachable/wearable PPNG is beneficial for body movement analysis during sports activities and shows its potential for use as an epidermal device for skin motion monitoring. ${ }^{44}$ 
(a)

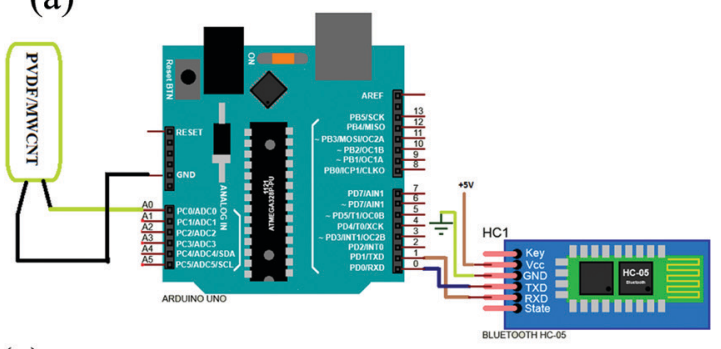

(b)

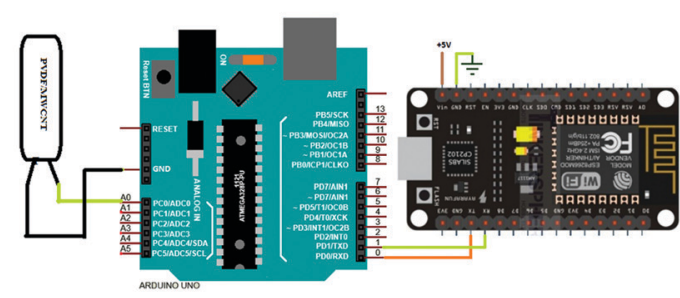

(c)

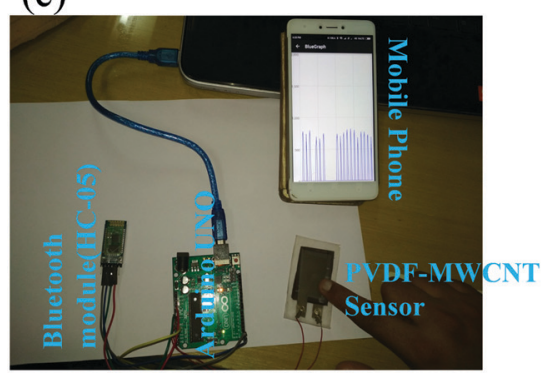

(d)

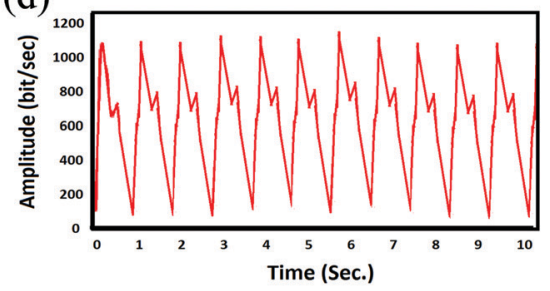

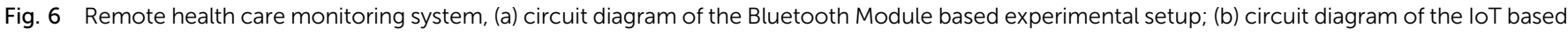

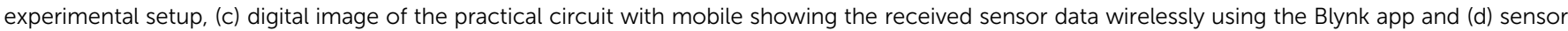
output response graph using finger imparting.

\section{Remote health monitoring}

Here we have demonstrated the real time practical application of the remote health monitoring system which is very much important for continuous monitoring of both virus suspected and infected patients, and reducing the risk of caregivers being exposed to the virus. We have designed two methods using a (i) Bluetooth Module and (ii) a Nodemcu Wi-Fi Module. Fig. 6a shows the basic circuit diagram of the experimental setup for using the Bluetooth Module and Fig. $6 \mathrm{~b}$ shows the basic circuit diagram of the experimental setup for using the IoT based remote health care monitoring system. In the first case a Bluetooth Module (HC-05), one Arduino UNO board and an Android mobile phone with the Blynk app are the main components, whereas in the second method, the main elements/ components are a single chip ESP8266A Wi-Fi module and a PC/Smart phone with the Blynk app. The single chip ESP8266A has $512 \mathrm{~KB}$ Flash memory and 10 bit ADC in set. It is run on L106, 32 bit MCU with a Real Time Operating System (RTOS). The clock speed is $80 \mathrm{MHz}$. Also it has $802.11 \mathrm{~b} / \mathrm{g} / \mathrm{h}$ and the frequency range is 2.4-2.5 G (2400-2483.5 M). With the help of MCU, we performed analog to digital conversion of the PPNG/sensor data value for processing. The waveforms are processed and the corresponding data are sent to a local server made by using the ESP8266A HTTP protocol. The patient or doctor can easily check the report on this network through WiFi/Bluetooth using their smart phones and laptops. Fig. 6c shows the real time practical circuit which shows the PPNG/sensor response under repeated finger touch in the smart phone screen through the local server for the IoT based remote health care monitoring system and Fig. 6d shows the plotted sensor output graph.

Using this system the clinician can monitor several physiological signals such as heartbeat, respiration rate, body temperature, coughing signal and so on of the virus infected/suspected patients even in a quarantine situation round the clock wirelessly without direct physical contact with the patient which simply prevents further spread of the virus. In future, the envisioned strategy through non-invasive piezo- and pyro-electric-based wearable sensors will be grown up for infectious diseases and in vivo body implantable. $^{45,46}$

\section{Conclusions}

In summary, we have developed a single device platform based on an all-fiber nanogenerator for harvesting both the mechanical and thermal energy using PVDF-MWCNT electrospun nanofibers as active piezo- and pyro-electric components respectively. It has been observed that the content of electroactive phases, degree of crystallinity and thermal stabilization of the $\beta$-phase within PVDF nanofibers were mainly affected by the synergistic effect of interfacial interactions and mechanical stretching forces exerted during the collection of fibers. As a consequence, the mechanically robust structure of PVDF-MWCNT nanofibers possesses an enhanced piezoelectric charge co-efficient $\left(\left|d_{33}\right| \sim\right.$ $51.3 \mathrm{pC} \mathrm{N}^{-1}$ ) and figure of merit (FoM $\left.\approx 5.95 \times 10^{-11} \mathrm{~Pa}^{-1}\right)$ in comparison to the neat PVDF nanofibers $\left(\left|d_{33}\right| \sim 22 \mathrm{pC} \mathrm{N}^{-1}\right.$ and FoM $\left.\approx 9.7 \times 10^{-12} \mathrm{~Pa}^{-1}\right)$. In addition to that the PPNG shows an ultra-fast response time of $\sim 10 \mathrm{~ms}$, high electrical throughput (output voltage $\sim 35 \mathrm{~V}$ and maximum power density $\sim 34 \mu \mathrm{W}$ $\mathrm{cm}^{-2}$ ) and power conversion efficiency $\left(\eta_{\text {piezo }} \sim 19.3 \%\right)$. As a result, the nanogenerator drives several consumer electronic components such as commercial capacitors and LEDs by simple human finger imparting. In addition, we have also developed a human health care monitoring system where our developed sensor can distinguish different muscle vibration signals from different parts of our body. Finally, a wireless health care system for remote health monitoring using IoT and a Bluetooth module 
with an Arduino Uno is developed by which a clinician can monitor several physiological signals of the virus infected/ suspected patients round the clock wirelessly without direct physical contact with the patient which simply prevents further spread of the virus. This type of healthcare system is urgently needed for frontline health staff during the current pandemic. Overall, the integrated device design, portable nature, and higher thermal and mechanical sensing capabilities of our developed all-fiber device could find possible applications in artificial electronic skin, prosthetic limbs, rehabilitation programs and other artificial intelligence applications in the near future to monitor dynamic tactile and strain information.

\section{Experimental section}

\section{Materials}

We used PVDF pellets ( $M_{\mathrm{w}}=275000$, Sigma-Aldrich, USA), acetone (Merck Chemical, India), $N, N$-dimethyl formamide (DMF from Merck) and MWCNT powder (Merck, India).

The remote healthcare system included an Arduino Uno which is an ATMega 328 microcontroller based system, an Arduino Uno based Wi-Fi Shield ESP8266MOD which is used as an IoT based gateway for establishment of communication with the IoT cloud and the system and a Bluetooth module (HC-05) and our proposed nanofiber based sensor.

\section{Fabrication of electrospun nanofibers}

The PVDF solution was prepared by dissolving $12 \mathrm{wt} \%$ of PVDF pellets in DMF/acetone $(60: 40 \mathrm{v} / \mathrm{v})$ solution under continuous stirring until the solution was acquired. Then $0.2 \mathrm{wt} \%(\mathrm{w} / \mathrm{v} \%)$ of MWCNTs was added to the resulting solution. The solution was stirred, followed by sonication in an ultra-sonication bath at room temperature until uniformly dispersed MWCNTs within the PVDF solution were obtained. Then, the resulting solution was placed in a plastic syringe $(15 \mathrm{~mL})$ tipped with a stainless steel needle with an inner diameter of $0.5 \mathrm{~mm}$. Electrospinning was carried out at $11 \mathrm{kV}$ from a high voltage power supply, where the stainless steel needle is connected to the positive terminal of the high voltage power supply and the metallic collector is grounded. A syringe pump was applied to deliver the solution into the needle tip at a flow rate of $0.5 \mathrm{ml} \mathrm{h}^{-1}$ and the electrospun nanofibers were collected through the aluminum-foil covered roller collector. The needle-to-roller collector distance of $12 \mathrm{~cm}$ was optimized. All experiments were supervised at room temperature and a relative humidity of $43 \pm 5 \%$. The schematic of the electrospinning set up used for the fabrication of the large area PVDF-MWCNT nanofiber mat is shown in Fig. S5 (ESI $\dagger$ ).

\section{Fabrication of the all-fiber PPNG}

The details of the fabrication process of our proposed all-fiber PPNG are discussed in the section "All-Fiber nanogenerator/ sensor fabrication strategy", under the Results and discussion part. The details of the device structure are shown in Fig. 1. Here the copper $(\mathrm{Cu})$-nickel $(\mathrm{Ni})$ plated interlocked micro-fiber based polyester fabric (supplied by Coatex Industries, India) was used as the top and bottom electrodes and the PVDFMWCNT nanofibers were used as the active layer. Finally the PPNG was further encapsulated with PDMS (Sylgard 184 silicone elastomer) for protection from the environment and to ensure a compact structure of the device. The complete thickness $(t)$ of the PPNG is $\sim 5 \mathrm{~mm}$ and the effective contact area of the PPNG is $7.5 \mathrm{~cm}^{2}$.

\section{Characterization}

To investigate in detail the surface morphology of the electrospun fibers, field emission scanning electron microscopy (FE-SEM, FEI, INSPECT F50) was undertaken at an acceleration voltage of $20 \mathrm{kV}$. The structural composition of the electrospun fibers was measured by Fourier transform infrared spectroscopy (FT-IR) (Bruker, Tensor II). X-ray diffraction (XRD) patterns of the electrospun nanofibers were recorded using an X-ray diffractometer (Bruker D8 Advance) with $\mathrm{CuK}_{\alpha}(\lambda \sim 1.5406 \AA)$ monochromatic radiation operated at $40 \mathrm{kV}$ and $30 \mathrm{~mA}$. Finally the output voltage response from the PPNG under different applied stress was recorded using a digital oscilloscope (Agilent DSO3102A) and current measurements were carried out using a Keithley 6485 picoammeter.

\section{Conflicts of interest}

The authors declare no competing financial interest.

\section{Acknowledgements}

This work was financially supported by a grant from the Science and Engineering Research Board (EEQ/2018/001130), Government of India. Kuntal Maity and Krittish Roy are thankful to the UGC (No. P-1/RS/319/14) and DST (IF160559) for providing the fellowship. Finally, we are grateful to Prof. Christopher Rhys Bowen for reading the entire manuscript and for providing valuable comments and corrections.

\section{References}

1 A. Kaur and A. Jasuja, Adv. Comput. Sci. Inf. Technol., 2017, 4, 80-84.

2 S. K. Ghosh, K. Roy, H. K. Mishra, M. R. Sahoo, B. Mahanty, P. N. Vishwakarma and D. Mandal, ACS Sustainable Chem. Eng., 2020, 8, 864-873.

3 H. He, T. Zhao, H. Guan, T. Zhong, H. Zeng, L. Xing, Y. Zhang and X. Xue, Sci. Bull., 2019, 64, 1409-1417.

4 H. Guan, T. Zhong, H. He, T. Zhao, L. Xing, Y. Zhang and X. Xue, Nano Energy, 2019, 59, 754-761.

5 Y. Zang, F. Zhang, C. Di and D. Zhu, Mater. Horiz., 2015, 2, 140-156.

6 Z. Liu, T. Zhao, H. Guan, T. Zhong, H. He, L. Xing and X. Xue, J. Mater. Sci. Technol., 2019, 35, 2187-2193. 
7 S. H. Lee, C. K. Jeong, G.-T. Hwang and K. J. Lee, Nano Energy, 2015, 14, 111-125.

8 R. G. Multhaupt, Ferroelectrics, 1987, 75, 385-396.

9 B. Mahanty, S. K. Ghosh, S. Garain and D. Mandal, Mater. Chem. Phys., 2017, 186, 327-332.

10 S. K. Ghosh, W. Rahman, T. R. Middya, S. Sen and D. Mandal, Nanotechnology, 2016, 27, 215401.

11 D. R. Dillon, K. K. Tenneti, C. Y. Li, F. Ko, I. Sics and B. S. Hsiao, Polymer, 2006, 47, 1678-1688.

12 K. Maity, B. Mahanty, T. K. Sinha, S. Garain, A. Biswas, S. K. Ghosh, S. Manna, S. K. Ray and D. Mandal, Energy Technol., 2017, 5, 234-243.

13 K. Park, S. Xu, Y. Liu, G.-T. Hwang, S.-J. L. Kang, Z. L. Wang and K. J. Lee, Nano Lett., 2010, 10, 4939-4943.

14 Y. M. Yousry, K. Yao, S. Chen, W. H. Liew and S. Ramakrishna, Adv. Electron. Mater., 2018, 4, 1700562.

15 D. Mandal, S. Yoon and K. J. Kim, Macromol. Rapid Commun., 2011, 32, 831-837.

16 L. Wang, Y. Wang, M. Wu, Z. Wei, C. Cui, M. Mao, J. Zhang, X. Han, Q. Liu and J. Ma, Small, 2018, 14, 1800737.

17 P. Martins, A. C. Lopes and S. L. Mendez, Prog. Polym. Sci., 2014, 39, 683-706.

18 B. Mahanty, S. K. Ghosh, S. Jana, K. Roy, S. Sarkar and D. Mandal, Sustainable Energy Fuels, 2021, 5, 1003-1013.

19 A. Biswas, K. Henkel, D. Schmeißer and D. Mandal, Phase Transitions, 2017, 19, 1205.

20 D. Chen, M. Wu, W. Wang and T. Liu, J. Macromol. Sci., Part B: Phys., 2010, 49, 1069-1082.

21 S. K. Ghosh, M. Xie, C. R. Bowen, P. R. Davies, D. J. Morgan and D. Mandal, Sci. Rep., 2017, 7, 16703.

22 S. K. Ghosh and D. Mandal, Nano Energy, 2018, 53, 245-257.

23 G. H. Kim, S. M. Hong and Y. Seo, Phys. Chem. Chem. Phys., 2009, 11, 10506-10512.

24 K. Roy, S. K. Ghosh, A. Sultana, S. Garain, M. Xie, C. R. Bowen, K. Henkel, D. Schmeißer and D. Mandal, ACS Appl. Nano Mater., 2019, 2, 2013-2025.

25 C. R. Bowen, H. A. Kim, P. M. Weaver and S. Dunnc, Energy Environ. Sci., 2014, 7, 25-44.

26 S. K. Ghosh, T. K. Sinha, M. Xie, C. R. Bowen, S. Garain, B. Mahanty, K. Roy, K. Henkel, D. Schmeißer, J. K. Kim and D. Mandal, ACS Appl. Electron. Mater., 2021, 3, 248-259.
27 Y. Yang, K. C. Pradel, Q. Jing, J. M. Wu, F. Zhang, Y. Zhou, Y. Zhang and Z. L. Wang, ACS Nano, 2012, 6, 6984-6989.

28 T. Zhao, W. Jiang, H. Liu, D. Niu, X. Li, W. Liu, X. Li, B. Chen, Y. Shi, L. Yin and B. Lu, Nanoscale, 2016, 8, 8111-8117.

29 C. Li, P.-M. Wu, S. Lee, A. Gorton, M. J. Schulz and C. H. Ahn, J. Microelectromech. Syst., 2008, 17, 334-341.

30 B. Wang, C. Liu, Y. Xiao, J. Zhong, W. Li, Y. Cheng, B. Hu, L. Huang and J. Zhou, Nano Energy, 2017, 32, 42-49.

31 A. Wang, M. Hu, L. Zhou and X. Qiang, Nanomaterials, 2018, 8, 1021-1032.

32 G. Zhao, X. Zhang, X. Cui, S. Wang, Z. Liu, L. Deng, A. Qi, X. Qiao, L. Li, C. Pan, Y. Zhang and L. Li, ACS Appl. Mater. Interfaces, 2018, 10, 15855-15863.

33 Y. R. Wang, J. M. Zheng, G. Y. Ren, P. H. Zhang and C. Xu, Smart Mater. Struct., 2011, 20, 045009.

34 W. Guo, C. Tan, K. Shi, J. Li, X. X. Wang, B. Sun, X. Huang, Y.-Z. Long and P. Jiang, Nanoscale, 2018, 10, 17751-17760.

35 T. Sharma, S.-S. Je, B. Gill and J. X. J. Zhang, Sens. Actuators, A, 2012, 177, 87-92.

36 S. M. Hosseini and A. A. Yousefi., Org. Electron., 2017, 50, 121-129.

37 Q.-L. Zhao, G.-P. He, J.-J. Di, W.-L. Song, Z.-L. Hou, P.-P. Tan, D.-W. Wang and M.-S. Cao, ACS Appl. Mater. Interfaces, 2017, 9, 24696-24703.

38 M. Sharma, V. Srinivas, G. Madras and S. Bose, RSC Adv., 2016, 6, 6251.

39 S. K. Ghosh and D. Mandal, ACS Sustainable Chem. Eng., 2017, 5, 8836-8843.

40 Z. H. Liu, C. T. Pan, L. W. Lin and H. W. Lai, Sens. Actuators, A, 2013, 193, 13-24.

41 S. K. Ghosh and D. Mandal, Nano Energy, 2016, 28, 356-365. 42 P. Piirila and A. R. A. Sovijarvi, Eur. Respir. J., 1995, 8, 1949-1956.

43 J.-A. Bachorowski, M. J. Smoski and M. J. Owren, J. Acoust. Soc. Am., 2001, 110, 1581-1597.

44 T. Ray, J. Choi, J. Reeder, S. P. Lee, A. J. Aranyosi, R. Ghaffari and J. A. Rogers, Curr. Opin. Biomed. Eng., 2019, 9, 47-56.

45 S. K. Ghosh and D. Mandal, J. Mater. Chem. A, 2021, 9, 1887-1909.

46 S. K. Ghosh, J. Park, S. Na, M. P. Kim and H. Ko, Adv. Sci., 2021, 2005010, DOI: 10.1002/advs.202005010. 